\title{
Risk Factors for 28-Day Mortality in a Surgical ICU: A Retrospective Analysis of 347 Cases
}

\begin{abstract}
Yuanyuan Zhang, ',2 jia Zhang,,3 Zhaoqing Du, ${ }^{2,4}$ Yifan Ren,, Jieming Nie, ${ }^{6}$ Zheng $\mathrm{Wu}^{3}{ }^{3}$ Yi Lv, ${ }^{2,3}$ Jianbin $\mathrm{Bi}^{2,7}$

Rongqian $\mathrm{Wu}$ iD $^{2}$

'Department of Pediatrics, The First Affiliated Hospital of Xi'an Jiaotong University, Xi'an, Shaanxi Province, People's Republic of China; ${ }^{2}$ National Local Joint Engineering Research Center for Precision Surgery \& Regenerative Medicine, Shaanxi Provincial Center for Regenerative Medicine and Surgical Engineering, The First Affiliated Hospital of Xi'an Jiaotong University, Xi'an, Shaanxi Province, People's Republic of China; ${ }^{3}$ Department of Hepatobiliary Surgery, The First Affiliated Hospital of Xi'an Jiaotong University, Xi'an, Shaanxi Province, People's Republic of China; ${ }^{4}$ Department of General Surgery, The Second Affiliated Hospital of Xi'an Jiaotong University, Xi'an, Shaanxi Province, People's Republic of China; ${ }^{5}$ Department of Hepatobiliary Surgery, Shaanxi Provincial People's Hospital, Xi'an, Shaanxi, People's Republic of China; ${ }^{6}$ Department of Internal Medicine, The Third Affiliated Hospital of Guangzhou Medical University, Guangzhou, Guangdong Province, People's Republic of China; ${ }^{7}$ Department of Oncology, The Second Affiliated Hospital of Xi'an Jiaotong University, Xi'an, Shaanxi Province, People's Republic of China
\end{abstract}

Correspondence: Rongqian Wu; Jianbin $\mathrm{Bi}$ National Local Joint Engineering Research Center for Precision Surgery \& Regenerative Medicine, First Affiliated Hospital of Xi'an Jiaotong University, 76 West Yanta Road, P.O. Box 124, Xi'an, Shaanxi Province, 71006I, People's Republic of China Tel +86298265 754l

Email rwu00I@mail.xjtu.edu.cn; bijianbin9I@I63.com

\author{
This article was published in the following Dove Press journal: \\ This article was published in the following Dove Press journal:
Risk Management and Healthcare Policy
}

\author{
ra
}

\begin{abstract}
Purpose: Advances in surgical techniques and intensive care over the past decades have significantly reduced the mortality rates of critically ill surgical patients. However, evaluations of risk factors associated with mortality in surgical intensive care units (ICUs) are limited. The aim of this study was to analyze the independent risk factors for 28-day mortality of surgical ICU patients.
\end{abstract}

Patients and Methods: The clinical data of adult patients who were admitted to the surgical ICU in the First Affiliated Hospital of Xi'an Jiaotong University from June 2013 to June 2017 were collected. Univariate and multivariable logistic regression analyses were performed to examine risk factors associated with 28-day mortality.

Results: A total of 347 patients were included in this analysis. The overall 28-day mortality rate was $32.6 \%$. The major causes of surgical ICU admission were gastrointestinal diseases $(46.7 \%)$, infection $(20.5 \%)$, trauma $(8.9 \%)$, respiratory diseases $(8.9 \%)$ and cardiovascular diseases $(6.6 \%)$. The univariate analysis showed age, total bilirubin, prothrombin time, international normalized ratio, arterial lactate level, APACHE II and SOFA score at ICU admission were significantly associated with 28-day mortality. In the multivariate analysis, however, age [Odds Ratio (OR): 2.899, 95\% CI: 1.427-5.890, P=0.003], hypertension [OR: 3.630, 95\% CI: 1.545-8.531, $\mathrm{P}=0.003$ ], platelet count [OR: $1.004,95 \% \mathrm{CI}: 1.001-1.007$, $\mathrm{P}=0.015]$, arterial lactate level [OR: 1.186, 95\% CI: 1.088-1.293, $\mathrm{P}<0.001$ ] and SOFA score [OR: $1.289,95 \% \mathrm{CI}: 1.131-1.469, \mathrm{P}<0.001]$ were identified as the independent risk factors for 28-day mortality of patients in the surgical ICU.

Conclusion: In patients admitted to the surgical ICU, age 65 and older, a high arterial lactate level and SOFA score at ICU admission were associated with increased 28-day mortality.

Keywords: critical care, surgical intensive care units, 28-day mortality, clinical characteristics

\section{Introduction}

The surgical intensive care unit (ICU) provides perioperative care to critically ill patients. Advances in surgical techniques and intensive care over the past decades have significantly reduced the mortality rates of critically ill surgical patients. ${ }^{1,2}$ However, the burden of critically ill surgical patients is increasing as more and more elderly patients seeking surgical treatment. ${ }^{3}$ A better understanding of risk factors associated with mortality of surgical ICU patients is of great importance for treatment evaluation, patient triage and resource allocation.

The Sequential Organ Failure Assessment (SOFA) score and the Acute Physiology and Chronic Health Evaluation II (APACHE II) score are two 
commonly used scoring systems for risk assessment in critically ill patients. However, their predictive power in surgical ICU patients varies in previous studies. ${ }^{4}$ Comorbidities such as hypertension, diabetes mellitus, cardiovascular and respiratory diseases are often associated with poor outcomes in critically ill patients. ${ }^{5,6}$ Due to improvement in the management of these conditions, however, several recent studies have indicated that serious comorbidities are not independent risk factors for mortality in the ICU. ${ }^{7,8}$ The elderly patients are expected to consume more ICU resources in the future. ${ }^{9}$ However, very few studies have specifically investigated the impact of age on outcomes of patients admitted to surgical ICU. ${ }^{9}$ An elevated lactate level is a poor indicator of survival in critically ill patients. ${ }^{10,11}$ However, whether it is an independent risk factor for mortality in surgical ICU remains unknown. Thus, there is an urgent need for evaluations of risk factors associated with mortality in surgical ICU.

Several previous studies have investigated risk factors associated with mortality in surgical ICUs. However, different studies appeared to have different conclusions. Toufen et al showed that infection is an independent risk factor for mortality in SICU. ${ }^{12}$ A study from Thailand suggested that the development of AKI is associated with higher mortality in elderly SICU patients. ${ }^{13}$ The First Affiliated Hospital of Xi'an Jiaotong University is located in northwest China, a relatively underdeveloped region of China. The surgical ICU has 15 beds. In this surgical ICU, patients are managed by critical care doctors with $24 / 7$ inhouse ICU coverage. The surgeons were usually consulted by the critical care doctors when the patients they operated on stayed in the surgical ICU. The aim of this study, therefore, was to analyze the independent risk factors for 28-day mortality of surgical ICU patients in northwest China.

\section{Materials and Methods}

\section{Patients}

This is a retrospective observational study. The cohort was composed of 347 adult patients who were admitted to the surgical ICU at the first affiliated hospital of Xi'an Jiaotong University from June 2013 to June 2017. Patients admitted to the surgical ICU for anesthesia recovery only or who underwent organ transplantation were excluded. Our study complied with the provisions of Declaration of Helsinki, and the protocol was approved by the Ethics Committee of the First Affiliated Hospital of
Xi'an Jiaotong University. The patient's informed written consent was waived due to the retrospective nature of this study. Patient data were anonymized and de-identified prior to analysis.

\section{Data Collection}

The electronic medical records of all patients were viewed retrospectively. The patients' age, sex, primary ICU diagnosis, coexisting conditions, vital symptom, vital signs, laboratory data, ICU care and clinical outcomes were collected in details. Laboratory data included the blood routine test, liver function test, renal function test, electrolyte, blood coagulation and arterial blood gas tests. The severity of disease was assessed using the Acute Physiology and Chronic Health Evaluation II (APACHE II) score and the Sequential Organ Failure Assessment (SOFA) score. The ICU mortality was recorded. After leaving the surgical ICU, the patients were followed for 28 days to record the 28-day mortality.

\section{Statistical Analysis}

The distribution of the continuous variables was checked for normality using the Kolmogorov-Smirnov test. Normally distributed variables were expressed as mean $\pm \mathrm{SD}$, and differences between the two groups were analyzed by two-tailed Student $t$-test. Nonnormally distributed variables were expressed as medians (interquartile range) and differences between the two groups were compared by Wilcoxon rank sum test. Categorical variables were expressed as absolute numbers and percent frequencies and differences between the two groups were compared by Chi-square test or Fisher's exact test. Univariate and multivariate analysis by logistic regression models were performed for the analysis of risk factors. All variables that were found to be significant or almost significant $(\mathrm{P}<0.20)$ in the univariate analysis were entered into a multivariate analysis. PASW (18.0) software (SPSS Inc., Chicago, Illinois, USA) was used for statistical analyses. A two-tailed $\mathrm{p}$ value $<0.05$ was considered statistically significant.

\section{Results}

\section{Demographic Data}

The clinical data of 347 patients admitted to the surgical ICU at our hospital between June 2013 and June 2017 were analyzed. The overall 28-day mortality rate was $32.6 \%$. Demographic data of survivors and nonsurvivors are shown in Table 1 . The average age of the patients was 
Table I Demographic Data of the Patients Admitted to the Surgical ICU

\begin{tabular}{|c|c|c|c|c|}
\hline Variables & Overall $(n=347)$ & Survivors $^{a}(n=234)$ & Nonsurvivors $^{b}(n=\mid 13)$ & $P$ value \\
\hline Age (years) & $60.88 \pm 18.01$ & $59.80 \pm 17.7 \mid$ & $61.13 \pm 16.3$ & 0.106 \\
\hline Sex (male/female) & $219 / 128$ & $146 / 88$ & $73 / 40$ & 0.689 \\
\hline \multicolumn{5}{|l|}{ Primary ICU diagnosis } \\
\hline Gastrointestinal, n (\%) & $162(46.69)$ & II 4 (48.72) & $48(42.48)$ & 0.275 \\
\hline Infection, n (\%) & $71(20.46)$ & $47(20.09)$ & $24(21.24)$ & 0.803 \\
\hline Trauma, n (\%) & $31(8.93)$ & $25(10.68)$ & $6(5.31)$ & 0.100 \\
\hline Respiratory, n (\%) & $31(8.93)$ & $20(8.55)$ & II (9.73) & 0.716 \\
\hline Cardiovascular, n (\%) & $23(6.63)$ & $10(4.27)$ & $13(11.50)$ & $0.011^{\#}$ \\
\hline Neurologic, n (\%) & $17(4.90)$ & II (4.70) & $6(5.31)$ & 0.805 \\
\hline Orthopedic, n (\%) & $7(2.02)$ & $3(1.28)$ & $4(3.54)$ & 0.161 \\
\hline Renal*, n (\%) & $5(1.44)$ & $4(1.71)$ & I $(0.88)$ & 0.546 \\
\hline \multicolumn{5}{|l|}{ Coexisting conditions } \\
\hline Smoking, n (\%) & $110(31.70)$ & $80(34.19)$ & $30(26.55)$ & 0.152 \\
\hline Chronic alcohol consumption, $\mathrm{n}(\%)$ & $80(23.05)$ & $59(25.21)$ & $21(18.58)$ & 0.169 \\
\hline Hypertension, n (\%) & $96(27.67)$ & $70(29.91)$ & $26(23.01)$ & 0.178 \\
\hline Diabetes mellitus, n (\%) & $44(12.68)$ & $28(11.97)$ & $16(14.16)$ & 0.565 \\
\hline Cirrhosis, n (\%) & $29(8.36)$ & $15(6.4 I)$ & 14 (I2.39) & 0.059 \\
\hline Ischemic heart disease, n (\%) & $33(9.5 \mathrm{I})$ & $19(8.12)$ & 14 (12.39) & 0.204 \\
\hline Stroke, n (\%) & $44(12.68)$ & $30(12.82)$ & 14 (I2.39) & 0.910 \\
\hline Malignant diseases, n (\%) & $73(21.04)$ & $45(19.23)$ & $28(24.78)$ & 0.235 \\
\hline COPD, n (\%) & $39(11.24)$ & $22(9.40)$ & $17(15.04)$ & 0.119 \\
\hline Surgery, n (\%) & $109(31.4 I)$ & $73(31.20)$ & $36(31.86)$ & 0.901 \\
\hline Gastrointestinal surgery, n (\%) & $26(7.49)$ & $17(7.26)$ & $9(7.96)$ & 0.817 \\
\hline Thoracic Surgery, n (\%) & $9(2.59)$ & $6(2.56)$ & $3(2.65)$ & 0.960 \\
\hline Hepatobiliary and pancreatic Surgery, n (\%) & $58(16.71)$ & $37(\mid 5.8 I)$ & $21(8.97)$ & 0.517 \\
\hline Neurosurgery, n (\%) & I (0.29) & $\mathrm{I}(0.43)$ & $0(0.00)$ & 0.486 \\
\hline Orthopedic surgery, n (\%) & $11(3.17)$ & $10(4.27)$ & I $(0.88)$ & 0.091 \\
\hline Urologic surgery, n (\%) & $2(0.58)$ & I $(0.43)$ & I $(0.88)$ & 0.598 \\
\hline Gynecologic surgery & $2(0.58)$ & $\mathrm{I}(0.43)$ & I (0.88) & 0.598 \\
\hline Emergency surgery, n (\%) & $16(4.61)$ & II (4.70) & $5(4.42)$ & 0.909 \\
\hline
\end{tabular}

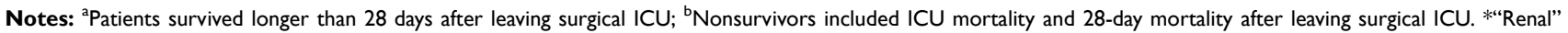
means acute kidney injury (AKI). ${ }^{\#} P$-value $<0.05$.

Abbreviation: COPD, chronic obstructive pulmonary disease.

$60.88 \pm 18.01$ years. The patients were predominantly males $(63.11 \%)$. However, there were no statistically significant differences in age and gender between survivors and nonsurvivors. The major causes of surgical ICU admission were gastrointestinal diseases $(46.7 \%)$, infection $(20.5 \%)$, trauma $(8.9 \%)$, respiratory diseases $(8.9 \%)$ and cardiovascular diseases $(6.6 \%)$. There were no major differences in causes for surgical ICU admission between survivors and nonsurvivors, except that the incidence of cardiovascular diseases was higher in nonsurvivors than that in survivors $(11.5 \%$ vs $4.27 \%, \mathrm{p}<0.05)$. In terms of coexisting conditions, there were no significant differences between survivors and nonsurvivors. Of the 347 patients, 109 underwent surgery, including 16 for emergency surgery. The other 238 patients were managed nonoperatively. There were no significant differences in the type of procedures between survivors and nonsurvivors.

\section{Clinical Characteristics}

The clinical characteristics of the patients are shown in Table 2. At surgical ICU admission, nonsurvivors were more likely to have jaundice and disturbance of consciousness than survivors. They also had higher levels of total bilirubin, prothrombin time, INR, arterial lactate, APACHE II and SOFA scores than survivors. There were no significant differences in other symptoms, signs and lab values at surgical ICU admission between the two groups. 
Table 2 Clinical Characteristics of the Patients Admitted to the Surgical ICU

\begin{tabular}{|c|c|c|c|c|}
\hline Variables & Overall $(n=347)$ & Survivors $(n=234)$ & Nonsurvivors $(n=|| 3)$ & $P$ value \\
\hline \multicolumn{5}{|l|}{ Symptoms at ICU admission } \\
\hline Fever, n (\%) & $34(9.80)$ & $25(10.68)$ & $9(7.96)$ & 0.425 \\
\hline Jaundice, n (\%) & $32(9.22)$ & $16(6.84)$ & $16(14.16)$ & $0.027^{\#}$ \\
\hline Hematemesis and hematochezia, $n$ (\%) & $12(3.46)$ & $7(2.99)$ & $5(4.42)$ & 0.493 \\
\hline Nausea and vomiting, n (\%) & $6(1.73)$ & $4(\mathrm{I} .7 \mathrm{I})$ & $2(1.77)$ & 0.968 \\
\hline Oliguria, n (\%) & $6(1.73)$ & $5(2.14)$ & I $(0.88)$ & 0.402 \\
\hline Disturbance of consciousness*, $\mathrm{n}(\%)$ & $89(25.65)$ & $45(19.23)$ & $44(38.94)$ & $<0.00 I^{\# \#}$ \\
\hline \multicolumn{5}{|l|}{ Signs at ICU admission } \\
\hline Body temperature $\left({ }^{\circ} \mathrm{C}\right)$ & $37.2 \pm 0.97$ & $37.2 \pm 0.89$ & $37.3 \pm 1.12$ & 0.136 \\
\hline Pulse rate (times per minute) & $104 \pm 26.2$ & $103 \pm 25.8$ & $107 \pm 27.7$ & 0.173 \\
\hline Respiratory rate (times per minute) & $24 \pm 8.6$ & $24 \pm 9.0$ & $25 \pm 7.9$ & 0.568 \\
\hline MAP $(\mathrm{mmHg})$ & $90 \pm 17.9$ & $91 \pm 16.9$ & $88 \pm 19.8$ & 0.101 \\
\hline \multicolumn{5}{|l|}{ Lab values at ICU admission } \\
\hline WBC $\left(10^{9} / \mathrm{L}\right)$ & $12.9 \pm 9.05$ & $12.9 \pm 9.53$ & $12.7 \pm 7.97$ & 0.823 \\
\hline $\operatorname{PLT}\left(10^{9} / \mathrm{L}\right)$ & $146.8 \pm 111.58$ & $153.3 \pm 112.44$ & $132.8 \pm 108.92$ & 0.112 \\
\hline ALT (U/L) & $186.8 \pm 504.05$ & $185.1 \pm 489.98$ & $233.4 \pm 569.65$ & 0.243 \\
\hline AST (U/L) & $289.3 \pm 934.09$ & $255.4 \pm 907.49$ & $361.6 \pm 988.98$ & 0.328 \\
\hline $\mathrm{TB}(\mu \mathrm{mol} / \mathrm{L})$ & $79.6 \pm 124.67$ & $64.0 \pm 102.40$ & $112.9 \pm 157.86$ & $0.001^{\# \#}$ \\
\hline Albumin $(\mathrm{g} / \mathrm{L})$ & $29.0 \pm 6.81$ & $29.4 \pm 7.12$ & $28.0 \pm 6.03$ & 0.085 \\
\hline PT (s) & $18.5 \pm 8.96$ & $|7.3 \pm 5.4|$ & $21.3 \pm 13.72$ & $<0.00 \mathrm{I}^{\# \#}$ \\
\hline INR & $1.6 \pm 0.91$ & $1.5 \pm 0.63$ & $1.9 \pm 1.31$ & $<0.00 I^{\# \#}$ \\
\hline Creatinine $(\mu \mathrm{mol} / \mathrm{L})$ & $127.0 \pm 131.52$ & $128.0 \pm 133.96$ & $124.8 \pm 126.66$ & 0.836 \\
\hline $\mathrm{Na}^{+}(\mathrm{mmol} / \mathrm{L})$ & $137.3 \pm 7.78$ & $136.9 \pm 8.70$ & $138.0 \pm 9.65$ & 0.218 \\
\hline $\mathrm{K}^{+}(\mathrm{mmol} / \mathrm{L})$ & $4.0 \pm 1.35$ & $3.9 \pm 0.71$ & $4.1 \pm 2.15$ & 0.417 \\
\hline $\mathrm{PaO}_{2} / \mathrm{FiO}_{2}{ }^{\mathrm{a}}$ & $3.4 \pm 2.92$ & $3.5 \pm 2.35$ & $3.1 \pm 3.88$ & 0.256 \\
\hline Arterial lactate $(\mathrm{mmol} / \mathrm{L})$ & $3.4 \pm 4.14$ & $2.4 \pm 2.76$ & $5.7 \pm 5.55$ & $<0.00 I^{\# \#}$ \\
\hline Procalcitonin $(\mathrm{ng} / \mathrm{mL})^{\mathrm{b}}$ & $16.1 \pm 36.22$ & $15.6 \pm 36.90$ & $17.4 \pm 34.49$ & 0.703 \\
\hline Positive blood bacterial culture ${ }^{c}, \mathrm{n}(\%)$ & $52(27.66)$ & $34(26.26)$ & $18(30.51)$ & 0.555 \\
\hline APACHE II at ICU admission & $16.39 \pm 7.61$ & $14.18 \pm 8.15$ & $20.96 \pm 8.31$ & $<0.00$ I\# \\
\hline SOFA score at ICU admission & $7.67 \pm 4.87$ & $8.18 \pm 3.57$ & $10.76 \pm 5.7 \mid$ & $<0.00 I^{\# \#}$ \\
\hline
\end{tabular}

Notes: a'Survivors $(n=214)$, nonsurvivors $(n=99)$; 'Survivors $(n=205)$, nonsurvivors $(n=76)$. "Survivors $(n=129)$, nonsurvivors $(n=59)$. * “Yes" in "disturbance of consciousness" was given when the patient has difficulty maintaining wakefulness and/or has impaired awareness of him/herself and his/her environment. ${ }^{\#} P$-value $<0.05,{ }^{\#} P$-value $<0.01$.

Abbreviations: HCT, hematocrit; WBC, white blood cell count; PLT, platelet count; ALT, alanine aminotransferase; AST, alanine aminotransferase; TB, total bilirubin; PT, prothrombin time; INR, international normalized ratio; $\mathrm{PaO}_{2} / \mathrm{FiO}_{2}$, oxygen partial pressure/oxygen concentration; MAP, mean arterial pressure; APACHE II, acute physiology and chronic health evaluation; SOFA score, sequential organ failure assessment score.

\section{Risk Factors of 28-Day Mortality by Univariate and Multivariate Analyses}

To investigate the association of patients' demographic data and clinical characteristics with 28-day mortality, we firstly conducted univariable logistic regression analysis. As shown in Table 3, patients' age, total bilirubin (TB), prothrombin time (PT), international normalized ratio (INR), arterial lactate, APACHE II and SOFA score at ICU admission were associated significantly with 28-day mortality of surgical ICU patients. Noticeably, gender, causes for ICU admission and coexisting conditions were not associated with 28-day mortality. We further conducted a multivariate logistic regression analysis using the parameters shown to be significant or almost significant $(\mathrm{P}<0.20)$ in the univariate analysis. As a result, age [Odds Ratio (OR): $2.899,95 \%$ CI: $1.427-5.890, \mathrm{P}=0.003$ ], hypertension [OR: $3.630,95 \% \mathrm{CI}: 1.545-8.531, \mathrm{P}=0.003$ ], platelet count [PLT, OR: $1.004,95 \%$ CI: $1.001-1.007$, $\mathrm{P}=0.015]$, arterial lactate level [OR: 1.186, 95\% CI: $1.088-1.293, \mathrm{P}<0.001]$ and SOFA score [OR: 1.289 , 95\% CI: $1.131-1.469, \mathrm{P}<0.001]$ at ICU admission were significantly associated with 28-day mortality of patients 
Table 3 Univariate and Multivariate Analyses of 28-Day Mortality

\begin{tabular}{|c|c|c|c|c|c|}
\hline \multirow[t]{2}{*}{ Parameters } & \multicolumn{2}{|c|}{ Univariate Analysis } & \multicolumn{3}{|c|}{ Multivariate Analysis } \\
\hline & OR $(95 \% \mathrm{Cl})$ & $P$ value & OR $(95 \% \mathrm{Cl})$ & $P$ value & VIF \\
\hline Age $(\geq 65 y /<65 y)$ & $1.627(1.036-2.557)$ & $0.035^{\#}$ & $2.899(I .427-5.890)$ & $0.003^{\# \#}$ & 1.300 \\
\hline Sex (male/female) & $1.100(0.689-1.756)$ & 0.690 & - & - & \\
\hline Smoking (yes/no) & $1.447(0.880-2.379)$ & 0.146 & $1.003(0.507-1.986)$ & 0.993 & 1.044 \\
\hline Chronic alcohol consumption (yes/no) & $1.477(0.845-2.581)$ & 0.171 & I. $148(0.5 \mid 5-2.562)$ & 0.736 & 1.079 \\
\hline Hypertension (yes/no) & $1.428(0.849-2.402)$ & 0.179 & $3.630(1.545-8.531)$ & $0.003^{\# \#}$ & 1.236 \\
\hline Diabetes mellitus (yes/no) & $0.824(0.426-1.594)$ & 0.565 & - & - & \\
\hline Cirrhosis (yes/no) & $0.484(0.225-1.042)$ & 0.064 & $0.558(0.185-1.683)$ & 0.300 & 1.222 \\
\hline Ischemic heart disease (yes/no) & $0.625(0.30 I-1.297)$ & 0.207 & - & - & \\
\hline Stroke (yes/no) & $1.040(0.528-2.049)$ & 0.910 & - & - & \\
\hline Malignant diseases (yes/no) & $0.723(0.423-1.236)$ & 0.236 & - & - & \\
\hline COPD & $0.586(0.298-1.154)$ & 0.122 & $0.907(0.348-2.367)$ & 0.842 & 1.188 \\
\hline Bacterial culture of blood (yes/no) $^{a}$ & $0.812(0.439-1.503)$ & 0.508 & - & - & - \\
\hline SOFA score at ICU admission & $1.310(1.222-1.405)$ & $<0.001^{\# \#}$ & $1.289(1.131-1.469)$ & $<0.00 I^{\# \#}$ & 3.234 \\
\hline APACHE II at ICU admission (w/o age)* & $1.139(1.099-1.181)$ & $<0.00 I^{\# \#}$ & $1.028(0.967-1.093)$ & 0.378 & 2.347 \\
\hline Surgery & $0.970(0.598-1.572)$ & 0.901 & - & - & \\
\hline Emergency surgery & $1.065(0.361-3.143)$ & 0.909 & - & - & \\
\hline PLT $\left(10^{9} / \mathrm{L}\right)$ & $0.998(0.996-1.000)$ & 0.114 & $1.004(1.001-1.007)$ & $0.015^{\#}$ & 1.357 \\
\hline WBC $\left(10^{9} / \mathrm{L}\right)$ & $0.997(0.972-1.023)$ & 0.822 & - & - & \\
\hline TB & $1.003(1.001-1.005)$ & $0.00 I^{\# \#}$ & $1.000(0.997-1.003)$ & 0.961 & 1.477 \\
\hline Creatinine & $1.000(0.998-1.002)$ & 0.836 & - & - & \\
\hline Albumin & $0.969(0.935-1.004)$ & 0.086 & $0.970(0.920-1.022)$ & 0.251 & 1.124 \\
\hline PT & $1.064(1.025-1.105)$ & $0.00 I^{\# \#}$ & $0.966(0.882-1.059)$ & 0.461 & 7.166 \\
\hline $\mathrm{Na}^{+}$ & $1.019(0.989-1.049)$ & 0.220 & - & - & \\
\hline $\mathrm{K}^{+}$ & $1.069(0.905-1.262)$ & 0.432 & - & - & \\
\hline INR & $1.742(1.256-2.415)$ & $0.001^{\# \#}$ & $1.210(0.438-3.340)$ & 0.713 & 7.045 \\
\hline $\mathrm{PaO}_{2} / \mathrm{FiO}_{2}{ }^{\mathrm{b}}$ & $0.940(0.843-1.048)$ & 0.265 & - & - & \\
\hline Arterial lactate & $1.253(1.156-1.358)$ & $<0.00 I^{\# \#}$ & $1.186(1.088-1.293)$ & $<0.00 I^{\# \#}$ & 1.301 \\
\hline Procalcitonin ${ }^{c}$ & $1.001(0.994-1.008)$ & 0.702 & - & - & \\
\hline Blood transfusion (yes/no) & $0.838(0.529-1.327)$ & 0.450 & - & - & \\
\hline
\end{tabular}

Notes: *Since age is a factor in calculating APACHE II scores, they cannot be used together with age in multivariable analysis. Therefore, APACHE II scores without age

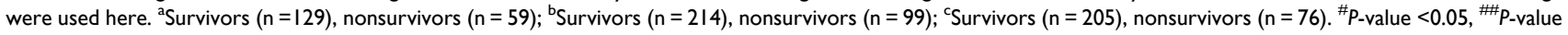
$<0.01$.

Abbreviations: COPD, chronic obstructive pulmonary disease; SOFA score, sequential organ failure assessment score; PLT, platelet count; WBC, white blood cell count; TB, total bilirubin; INR, international normalized ratio; $\mathrm{PaO}_{2} / \mathrm{FiO}_{2}$, oxygen partial pressure/oxygen concentration.

in the surgical ICU. However, as the OR for PLT was so close to 1 , the clinical significance of PLT for predicting mortality in a surgical ICU appeared to be very limited.

\section{Clinical Outcomes for Young and Elderly SICU Patients}

The clinical outcomes for young and elderly SICU patients are summarized in Table 4. The maximum APACHE II (without the age point) and SOFA score during their ICU stay as well as at discharge exhibited no differences between the young and elderly. The most common complications were multiple organ dysfunction syndrome
(MODS), acute kidney injury (AKI), sepsis and acute respiratory distress syndrome (ARDS), accounting for $16.14 \%, 15.27 \%, 12.39 \%$ and $11.24 \%$, respectively. The elderly were more likely to develop septic shock $(0.10 \%$ vs $7.69 \%, \mathrm{p}=0.002)$, ARDS (6.28\% vs $17.31 \%, \mathrm{p}=0.001)$ and acute coronary syndrome (ACS, $2.62 \%$ vs 17.95 , $\mathrm{p}=<0.001)$ than the young. The length of hospital and ICU stay as well as the incidence of ICU re-admission showed no difference between the two groups. The elderly patients had a slightly higher ICU mortality rate than the young patients. However, the difference did not reach statistical significance. Consistent with results in Table 3, 
Table 4 Clinical Outcomes for Young and Elderly SICU Patients

\begin{tabular}{|c|c|c|c|c|}
\hline Variables & Overall $(n=347)$ & The Young $(<65 y, n=|9|)$ & The Elderly $(\geq 65 y, n=\mid 56)$ & $P$ value \\
\hline Maximum APACHE II & $18.53 \pm 8.56$ & $16.97 \pm 8.20$ & $20.44 \pm 8.64$ & $<0.001^{\# \#}$ \\
\hline Maximum APACHE II (w/o age) & $13.20 \pm 9.34$ & $13.02 \pm 9.30$ & $13.42 \pm 9.40$ & 0.691 \\
\hline Maximum SOFA score & $8.75 \pm 5.66$ & $8.42 \pm 6.00$ & $9.158 \pm 5.21$ & 0.237 \\
\hline APACHE II at discharge & $16.61 \pm 9.64$ & $14.70 \pm 9.45$ & $18.9 \pm 9.38$ & $<0.00 I^{\# \#}$ \\
\hline APACHE II at discharge (w/o age) & $15.12 \pm 8.35$ & $15.29 \pm 8.10$ & $14.90 \pm 8.67$ & 0.670 \\
\hline SOFA score at discharge & $8.44 \pm 5.79$ & $8.06 \pm 6.08$ & $8.91 \pm 5.40$ & 0.173 \\
\hline \multicolumn{5}{|l|}{ Complications } \\
\hline Sepsis, n (\%) & 43 (12.39) & $24(12.57)$ & $19(12.18)$ & 0.914 \\
\hline Sepsis shock, n (\%) & $14(4.03)$ & $2(0.10)$ & $12(7.69)$ & $0.002^{\# \#}$ \\
\hline AKI, n (\%) & $53(15.27)$ & $30(|5.7|)$ & $23(14.74)$ & 0.804 \\
\hline ALI, n (\%) & $32(9.22)$ & $21(10.99)$ & II (7.05) & 0.207 \\
\hline ARDS, n (\%) & 39 (1 I.24) & $12(6.28)$ & $27(\mid 7.31)$ & $0.00 I^{\# \#}$ \\
\hline ACS, n (\%) & $33(9.5 \mathrm{I})$ & $5(2.62)$ & $28(17.95)$ & $<0.00 I^{\# \#}$ \\
\hline DIC, n (\%) & $5(1.44)$ & $3(1.57)$ & $2(1.28)$ & 0.822 \\
\hline MODS, n (\%) & $56(16.14)$ & $27(14.14)$ & $29(18.59)$ & 0.262 \\
\hline Length of hospital stay (days) & $14.12 \pm 14.38$ & $14.72 \pm 14.30$ & $14.39 \pm 14.48$ & 0.392 \\
\hline Length of ICU stay (days) & $10.85 \pm 12.82$ & $11.91 \pm 13.34$ & $9.56 \pm 12.08$ & 0.091 \\
\hline ICU re-admission, n (\%) & $48(13.83)$ & $22(\mathrm{I} I .52)$ & $26(16.66)$ & 0.167 \\
\hline ICU mortality, n (\%) & 61 (17.58) & $29(15.18)$ & $32(20.5 I)$ & 0.194 \\
\hline 28-day mortality, n (\%) & $113(32.56)$ & $53(27.75)$ & $60(38.46)$ & $0.034^{\#}$ \\
\hline
\end{tabular}

Notes: ${ }^{2} 28$-day overall mortality included ICU mortality and 28-day mortality after leaving ICU. ${ }^{\#} P$-value $<0.05$, ${ }^{\#} P$-value $<0.0$ I.

Abbreviations: APACHE II, acute physiology and chronic health evaluation; SOFA score, sequential organ failure assessment score; AKI, acute kidney injury; ALI, acute liver injury; ARDS, acute respiratory distress syndrome; ACS, acute coronary syndrome; DIC, disseminated intravascular coagulation; MODS, multiple organ dysfunction syndrome; ICU, intensive care unit.

the 28-day overall mortality was significantly higher in the elderly group than the young group $(38.46 \%$ vs $27.75 \%$, $\mathrm{p}=0.034)$.

\section{Discussion}

In this study, we identified four independent risk factors for 28-day mortality of patients in the surgical ICU: age 65 or older, hypertension, a high arterial lactate level and a high SOFA score at ICU admission. Although platelet count (PLT) was also significantly associated with 28-day mortality of surgical ICU patients in the multivariate logistic regression analysis, the OR for PLT was so close to 1. The clinical significance of PLT for predicting mortality in a surgical ICU appeared to be very limited. These findings would be useful for treatment evaluation, patient triage and resource allocation in surgical ICUs.

Aging affects many aspects of immune responses and is associated with an increasing vulnerability to many diseases and to death. ${ }^{14-17}$ Our current finding on the impact of aging on ICU mortality was consistent with several previous reports. ${ }^{18,19}$ Immunosenescence in the elderly contributes to the dysregulation of inflammatory responses. $^{20}$ Age-related changes in mitochondria and telomeres cause deterioration in cellular structure and function. ${ }^{21}$ As a result of inactivity, functional limitation, and poor or restricted diets, malnutrition is commonplace in elderly patients. ${ }^{22}$ Malnutrition can disrupt the function of various immune system components, weaken immune defense, and make elderly people vulnerable to various diseases. ${ }^{23}$ Sepsis, ARDS and ACS are common complications of ICU patients. Older age is an independent risk factor of sepsis. ${ }^{24}$ The incidence of sepsis increases with increasing age. ${ }^{25}$ In the current study, we also found the elderly patients were more likely to suffer other underlying diseases such as hypertension, diabetes mellitus and stroke. The elderly ICU patients had higher risks of developing complications including septic shock, ARDS and ACS. They also had a higher mortality rate. Taken together, these results suggest that compared with young surgical ICU patients, elderly surgical ICU patients were more likely to develop lifethreatening complications. The prevention and treatment of age-related diseases is a crucial task for the modern medical. 
Lactate and SOFA score mainly reflects the progress and transformation of the disease in real time. Elevated lactate levels indicate some level of shock or tissue malperfusion and have been found to be associated with worse outcomes. ${ }^{26}$ Excessive lactate production may give rise to lactic acidosis. However, it is probably not the elevated lactate per se, but the underlying condition that increases the risk for adverse outcomes. ${ }^{11}$ The SOFA score is an effective method to assess organ dysfunction in critically ill patients. ${ }^{27}$ It has been demonstrated that the SOFA score is a useful predictor of medical ICU mortality. ${ }^{28}$ In the current study, the univariate and multivariate analyses by logistic regression models also indicate SOFA scores were one of the independent risk factors for mortality of patients in the surgical ICU.

APACHE II is a severity of disease classification system consisting of acute physiology points, age points and chronic health points. ${ }^{29}$ It is widely used for severity evaluation for patients in the ICU. ${ }^{30}$ Since the purpose of this study was to investigate the impact of age on surgical ICU outcomes, we calculated the APACHE II score both with and without the age point. The results showed that although old ICU patients had higher overall APACHE II scores than young ones, the differences between the two groups became no significant when the age point were excluded from the calculation. More importantly, the multivariate analysis reveals that APACHE II scores both with (data not shown) and without the age point were not an independent risk factor for mortality of patients in the surgical ICU. This is not surprising. Several other studies have also shown the APACHE II score was not reliable in predicting mortality in the ICU. ${ }^{31,32}$ Although there are studies suggesting APACHE II could be used to predict post-ICU mortality, ${ }^{33}$ the predicted mortality of an APACHE II score is dependent on ICU admission indications. ${ }^{30}$ This discrepancy limits the application of the APACHE II score in a patient population with a variety of disease states, which is the case in our current study. Taken together, our data suggest that SOFA has greater prognostic accuracy for mortality than APACHE II in the surgical ICU. APACHE II may have limited utility for predicting mortality in a surgical ICU setting.

Some limitations need to be noted in the present study. Due to the lack of information, we were unable to calculate the patients' Glasgow Coma Scale (GCS) and body mass index (BMI) in this study. During our study period, there were about 400 patients who underwent organ transplantation in our hospital. The majority of organ transplant patients were younger than 65 years old, and their short-term mortality was relatively low compared with other SICU patients. Due to the unique nature of organ transplant patients, it would confound our analysis. Therefore, we decided to exclude them from the analysis. Like most ICUs, our surgical ICU has a mixed population. The primary ICU diagnosis was organized according to the organ-system in this study. In addition, this study was designed as a single-center retrospective cohort study. Therefore, it might not be effectively controlled and could be subjected to a selection bias, recall bias and some residual confounding. A multiple-center data were needed to validate the effect of age on the surgical ICU patients. Furthermore, this study was only concentrated on the short-term outcomes. This is due to the fact that the young and elderly ICU patients had significantly different underlying conditions, which may affect the long-term outcomes. In the future, a prospective propensity score-matched study is warranted to investigate for the longterm outcomes of surgical ICU patients.

\section{Conclusion}

In patients admitted to the surgical ICU, age 65 or older, hypertension, a high arterial lactate level and SOFA score at ICU admission were associated with increased 28-day mortality.

\section{Acknowledgments}

This project was supported by grants from the National Natural Science Foundation of China (No. 81770491), the Innovation Capacity Support Plan of Shaanxi Province (No. 2020TD-040), the Key R\&D Program of Shaanxi Province (No. 2021SF-002) and the Science Foundation of First Affiliated Hospital of Xi'an Jiaotong University (No. XJTU1AF-CRF-2020-025 and 2018QN-12).

\section{Disclosure}

The authors report no conflicts of interest in this work.

\section{References}

1. Darmon M, Bourmaud A, Georges Q, et al. Changes in critically ill cancer patients' short-term outcome over the last decades: results of systematic review with meta-analysis on individual data. Intensive Care Med. 2019;45:977-987. doi:10.1007/s00134-019-05653-7

2. van Breugel JMM, Niemeyer MJS, Houwert RM, et al. Global changes in mortality rates in polytrauma patients admitted to the ICU-a systematic review. World J Emerg Surg. 2020;15:55. doi:10.1186/s13017-020-00330-3

3. Shchatsko A, Purcell LN, Tignanelli CJ, et al. The effect of organ system surgery on intensive care unit mortality in a cohort of critically Ill Surgical patients. Am Surg. 2020:3134820956353. doi:10.1177/ 0003134820956353 
4. Hsu Y-T, He Y-T, Ting C-K, et al. Administrative and claims data help predict patient mortality in intensive care units by logistic regression: a Nationwide Database Study. Biomed Res Int. 2020;2020:9076739. doi:10.1155/2020/9076739

5. Jentzer JC, van Diepen S, Barsness GW, et al. Changes in comorbidities, diagnoses, therapies and outcomes in a contemporary cardiac intensive care unit population. Am Heart J. 2019;215:12-19. doi:10.1016/j.ahj.2019.05.012

6. Du Z, Zhou X, Zhao J, et al. Effect of diabetes mellitus on short-term prognosis of 227 pyogenic liver abscess patients after hospitalization. BMC Infect Dis. 2020;20(1):145. doi:10.1186/s12879-020-4855-9

7. Fernando SM, Mathew R, Hibbert B, et al. New-onset atrial fibrillation and associated outcomes and resource use among critically ill adults - a multicenter retrospective cohort study. Crit Care. 2020;24 (1):15. doi:10.1186/s13054-020-2730-0

8. Ogawa $\mathrm{T}$, Inoue $\mathrm{S}$, Inada $\mathrm{M}$, et al. Postoperative intensive care unit admission does not affect outcomes in elective surgical patients with severe comorbidity. Med Intensiva. 2020;44(4):216-225. doi:10.1016/j.medin.2019.01.003

9. Vallet H, Schwarz GL, Flaatten H, et al. Mortality of older patients admitted to an ICU: a systematic review. Crit Care Med. 2021;49 (2):324-334. doi:10.1097/CCM.0000000000004772

10. Chen X, Bi J, Zhang J, et al. The impact of serum glucose on the predictive value of serum lactate for hospital mortality in critically Ill surgical patients. Dis Markers. 2019;2019:1578502. doi:10.1155/ 2019/1578502

11. Chebl RB, Tamim H, Dagher GA, et al. Serum lactate as an independent predictor of in-hospital mortality in intensive care patients. $J$ Intensive Care Med. 2020;35(11):1257-1264. doi:10.1177/ 0885066619854355

12. Toufen C Jr, Franca SA, Okamoto VN, et al. Infection as an independent risk factor for mortality in the surgical intensive care unit. Clinics. 2013;68(8):1103-1108. doi:10.6061/clinics/2013(08)07

13. Trongtrakul K, Poopipatpab S, Pisitsak C, et al. Acute kidney injury in elderly patients in Thai-Surgical Intensive Care Units (THAI-SICU) Study. J Med Assoc Thai. 2016;99(Suppl 6):S209S218.

14. Agrawal A, Gupta S. Impact of aging on dendritic cell functions in humans. Ageing Res Rev. 2011;10(3):336-345. doi:10.1016/j. arr.2010.06.004

15. Wu R, Zhou M, Dong W, et al. Ghrelin hyporesponsiveness contributes to age-related hyperinflammation in septic shock. Ann Surg. 2009;250(1):126-133. doi:10.1097/SLA.0b013e3181ad85d6

16. Martin S, Perez A, Aldecoa C. Sepsis and Immunosenescence in the elderly patient: a review. Front Med. 2017;4:20. doi:10.3389/ fmed.2017.00020

17. Zhang J, Du Z, Bi J, et al. Comparison of clinical characteristics and outcomes of pyogenic liver abscess patients $<65$ years of age versus $>/=65$ years of age. BMC Infect Dis. 2019;19:233.

18. Level C, Tellier E, Dezou P, et al. Outcome of older persons admitted to intensive care unit, mortality, prognosis factors, dependency scores and ability trajectory within 1 year: a prospective cohort study. Aging Clin Exp Res. 2018;30(9):1041-1051. doi:10.1007/s40520-017-0871-z
19. Vargas N, Tibullo L, Landi E, et al. Caring for critically ill oldest old patients: a clinical review. Aging Clin Exp Res. 2017;29(5):833-845. doi:10.1007/s40520-016-0638-y

20. Macaulay R, Akbar AN, Henson SM. The role of the $\mathrm{T}$ cell in age-related inflammation. Age. 2013;35(3):563-572. doi:10.1007/ s11357-012-9381-2

21. Chistiakov DA, Sobenin IA, Revin VV, et al. Mitochondrial aging and age-related dysfunction of mitochondria. Biomed Res Int. 2014;2014:238463. doi:10.1155/2014/238463

22. Shpata V, Ohri I, Nurka T, et al. The prevalence and consequences of malnutrition risk in elderly Albanian intensive care unit patients. Clin Interv Aging. 2015;10:481-486. doi:10.2147/CIA.S77042

23. Pae M, Wu D. Nutritional modulation of age-related changes in the immune system and risk of infection. Nutr Res. 2017;41:14-35. doi:10.1016/j.nutres.2017.02.001

24. Martin GS, Mannino DM, Moss M. The effect of age on the development and outcome of adult sepsis. Crit Care Med. 2006;34 (1):15-21. doi:10.1097/01.CCM.0000194535.82812.BA

25. Starr ME, Saito H. Sepsis in old age: review of human and animal studies. Aging Dis. 2014;5:126-136. doi:10.14336/AD.2014.0500126

26. Zhang $\mathrm{Z}, \mathrm{Xu} \mathrm{X}$. Lactate clearance is a useful biomarker for the prediction of all-cause mortality in critically ill patients: a systematic review and meta-analysis*. Crit Care Med. 2014;42 (9):2118-2125. doi:10.1097/CCM.0000000000000405

27. Vincent JL, de Mendonca A, Cantraine F, et al. Use of the SOFA score to assess the incidence of organ dysfunction/failure in intensive care units: results of a multicenter, prospective study. Working group on "sepsis-related problems" of the European Society of Intensive Care Medicine. Crit Care Med. 1998;26:1793-1800. doi:10.1097/ 00003246-199811000-00016

28. Raith EP, Udy AA, Bailey M, et al. Prognostic accuracy of the SOFA score, SIRS criteria, and qSOFA score for in-hospital mortality among adults with suspected infection admitted to the intensive care unit. JAMA. 2017;317(3):290-300. doi:10.1001/ jama.2016.20328

29. Salluh JI, Soares M. ICU severity of illness scores: APACHE, SAPS and MPM. Curr Opin Crit Care. 2014;20:557-565. doi:10.1097/ MCC.0000000000000135

30. Niewinski G, Starczewska M, Kanski A. Prognostic scoring systems for mortality in intensive care units-the APACHE model. Anaesthesiol Intensive Ther. 2014;46:46-49. doi:10.5603/ AIT.2014.0010

31. Desai S, Lakhani JD. Utility of SOFA and APACHE II score in sepsis in rural set up MICU. J Assoc Physicians India. 2013;61:608-611.

32. Rapsang AG, Shyam DC. Scoring systems in the intensive care unit: a compendium. Indian J Crit Care Med. 2014;18(4):220-228. doi:10.4103/0972-5229.130573

33. Lee H, Lim CW, Hong HP, et al. Efficacy of the APACHE II score at ICU discharge in predicting post-ICU mortality and ICU readmission in critically ill surgical patients. Anaesth Intensive Care. 2015;43:175-186. doi:10.1177/0310057X1504300206
Risk Management and Healthcare Policy

\section{Publish your work in this journal}

Risk Management and Healthcare Policy is an international, peerreviewed, open access journal focusing on all aspects of public health, policy, and preventative measures to promote good health and improve morbidity and mortality in the population. The journal welcomes submitted papers covering original research, basic science, clinical \& epidemiological studies, reviews and evaluations, guidelines, expert opinion and commentary, case reports and extended reports. The manuscript management system is completely online and includes a very quick and fair peer-review system, which is all easy to use. Visit http://www.dovepress.com/testimonials.php to read real quotes from published authors. 